\title{
NÍVEIS DE NITRITO E NITRATO EM SALSICHAS COMERCIALIZADAS NA REGIÃO METROPOLITANA DO RECIFE ${ }^{1}$
}

\author{
Artur Bibiano de MELO FILHO ${ }^{2}$, Telma Maria Barreto BISCONTINI ${ }^{2, *}$,
}

\author{
Sâmara Alvachian Cardoso ANDRADE ${ }^{3}$
}

\begin{abstract}
RESUMO
Os sais de nitrato e nitrito em excesso prejudicam a saúde. Com o objetivo de avaliar a qualidade de salsichas comercializadas em Recife-PE, os niveis de nitrito residual e nitrato foram determinados em 54 amostras de salsichas, coletadas segundo região de origem e marca, de acordo com 3 grupos e 9 sub-grupos: $A)$ Sul $\left(A_{1}, A_{2}, A_{3}\right)$; B) Nordeste $\left(B_{1}, B_{2}\right.$, $\left.B_{3}\right)$, coletadas em supermercados; e, C) indústrias locais e sem marcas $\left(\mathrm{C}_{1}, \mathrm{C}_{2}, \mathrm{C}_{3}\right)$, em feiras livres. Aplicou-se testes ANOVA e Tukey. Constatou-se que no grupo (C) $67 \%$ das amostras apresentavam-se com nitrito residual $>150 \mathrm{mg} / \mathrm{kg}$ (18\% do total); e os níveis de nitrato $>300 \mathrm{mg} / \mathrm{kg}$ em 17(A), 67(B) e 83\%(C) das amostras $(\mathrm{p}<0,05)$, acima do nivel máximo permitido. Os resultados permitiram indicar que os níveis de nitrato e nitrito em salsichas representam um risco potencial à saúde do consumidor devido ao não cumprimento da legislação na produção e comercialização desse produto. Palavras-chave: nitrito; nitrato; salsichas; legislação.
\end{abstract}

\section{SUMMARY}

LEVEL NITRITE AND NITRATE IN SAUSAGES COMMERCIALIZED IN METROPOLITAN REGION OF RECIFE. The nitrite and nitrate salts in excess are harmfull to the health. With the objective to evaluate the quality of sausages commercialized in the Metropolitan Region of Recife, PE, the residual levels of nitrite and nitrate were measured in a 54 samples of hot-dog type sausage, that had constituted, as region of origin and mark, 3 groups and 9 sub-groups: (A) the south one (A1, A2, A3); B) northeast (B1, B2, B3), both, collected in supermarkets; and, C) local industries, without marks (C1, C2, C3), in free fairs. ANOVA and Tukey tests were applied. The results showed that in $67 \%$ of the sausages from free fairs (C), (18\% of total), the residual nitrite average were $>150 \mathrm{mg} / \mathrm{kg}$ above of the allowed maximum limit; and for nitrate value average, in $17(\mathrm{~A}), 67(\mathrm{~B})$ and $83 \%(\mathrm{C})$ of sausages, were $>300 \mathrm{mg} / \mathrm{kg}(\mathrm{max}$. allowed) (p< 0.05$)$. The results had allowed to conclude that the nitrite residual and nitrate levels in sausages, represent a risk to the health of the consumers due not following to the legislation in the production and commercialization of this product.

Keywords: nitrite; nitrate; sausages; legislation.

\section{1 - INTRODUÇÃO}

O mercado de embutidos tem apresentado significativa expansão e alta competitividade na última década, uma vez que o consumo de produtos cárneos como salsichas, lingüiças, mortadelas, hambúrgueres e outros, tornou-se parte do hábito alimentar de uma parcela considerável de consumidores brasileiros [12]. A fabricação de embutidos representa um importante segmento da industrialização de carnes; aproximadamente 1,2 milhões de toneladas foram produzidos no Brasil, em 1998 [18]. As salsichas representam o mais vendido produto cárneo industrializado, cuja produção atingiu 25.000ton em 1986, evoluindo para alcançar em 1993 uma cifra de 55.000ton. [7], cuja estimativa da indústria, aponta para um aumento de $45 \%$ nos anos de 1994 e 1995 [15].

O preço acessivel de algumas marcas, a praticidade do preparo e o valor protéico desses produtos, especialmente da salsicha, contribuem, para a redução do "déficit” nutricional, principalmente da população de menor renda. Todavia, convém considerar os principais diferenciadores entre os fabricantes: a qualidade, o preço e a apresentação do produto.

\footnotetext{
Recebido para publicação em 10/02/2003. Aceito para publicação em $31 / 05 / 2004$ (001064).

2. Departamento de Nutrição, UFPE - Av. Moraes Rego, Cidade Universitária, Recife-PE, Brasil. E-mail: bibiano@nutricao.ufpe.br; bisconti@npd.ufpe.br 3. Departamento de Engenharia Química, UFPE - Av. Moraes Rego, Cidade Universitária, Recife, $P E$, Brasil

* A quem a correspondência deve ser enviada.
}

Entretanto, observa-se a comercialização de produtos embutidos de marcas desconhecidas, elaborados artesanalmente, sem qualquer orientação ou fiscalização, por parte dos órgãos competentes, oferecidos indiscriminadamente, inclusive em feiras livres, expondo os consumidores aos riscos inerentes à ingestão de alimentos processados em condições precárias, ressaltando-se os relacionados aos aditivos empregados.

É secular o emprego de sais de nitrito e nitrato de sódio ou potássio em produtos embutidos de carne. Segundo alguns pesquisadores [1, 6, 13], a utilização desses sais tem por finalidade conferir cor e sabor aos produtos, além de funcionar como agente antimicrobiano e antioxidante [4]. SILVA [14] relata que o nitrito de sódio tem a capacidade de inibir o crescimento e a produção de toxina das várias espécies de Clostridium.

A aplicação desses sais acima do limite máximo estabelecido pela legislação vigente pode acarretar sérios riscos à saúde humana, pela possibilidade de manifestações de efeitos tóxicos agudos e crônicos. O nitrito ingerido em excesso pode agir sobre a hemoglobina e originar a metahemoglobinemia, impedindo que ela exerça a função normal de transportar oxigênio [8]. A reação do íon nitrito com aminas e amidas presentes no meio pode dar origem às nitrosaminas e nitrosamidas, substâncias consideradas carcinogênicas, mutagênicas e teratogênicas [5].

Quanto ao nitrato, é reduzido a nitrito por enzimas produzidas por microrganismos (micrococcus) cuja proliferação é favorecida por manuseio e processamento 
inadequado dos alimentos. As condições ácidas do estômago também promovem a redução do nitrato a nitrito, favorecendo igualmente a metahemoglobinemia [11].

Alguns autores relatam que derivados de carne de fabricação nacional apresentam, na maioria das vezes, níveis de nitrito e nitrato acima do permitido pela legislação [9, 10, 16]. Em países de clima tropical, em que as altas temperaturas e a umidade favorecem o ataque microbiano, esses aditivos contribuem, de forma significativa, para a conservação de embutidos. Contudo, tendo em vista os riscos associados ao emprego indiscriminado desses aditivos, sua utilização deve ser devidamente inspecionada.

A adição de nitrito e nitrato em alimentos é oficialmente regulamentada, na maioria dos países. Contudo, as orientações quanto ao seu emprego têm sofrido alterações nos últimos anos, principalmente nos países em desenvolvimento. No Brasil, até dezembro de 1998 era permitido um limite máximo de 200 e $500 \mathrm{mg} / \mathrm{kg}$, respectivamente, reduzindo, a partir daquela data, para valores de 150 e $300 \mathrm{mg} / \mathrm{kg}$ [3], valores limites, considerados elevados por alguns autores, uma vez que em outros países, a legislação estabelece valores inferiores [19].

Apesar da ampliação e surgimento de novos produtos cárneos lançados no mercado nesses últimos anos, há escassez de informações sobre os teores de nitrito residual e nitrato, principalmente em salsichas.

A proposta do presente trabalho foi avaliar a qualidade de salsichas comercializadas na Região Metropolitana do Recife verificando o nível de adequação à legislação quanto aos teores de nitrito residual e nitrato em salsichas, visando detectar os níveis de segurança alimentar para o consumidor.

\section{2 - MATERIAL E MÉTODO}

Na Região Metropolitana do Recife foram coletadas, em diferentes pontos de comercialização, 54 amostras de salsichas tipo hot-dog, agrupadas segundo a região de origem e marcas em 3 grupos e 9 sub-grupos: A) Sul do país $\left(A_{1}, A_{2}, A_{3}\right)$; B) região Nordeste $\left(B_{1}, B_{2}, B_{3}\right)$, adquiridas em supermercados e produzidas com inspeção federal; e, C) indústrias locais, sem marcas e inspeção definidas $\left(\mathrm{C}_{1}, \mathrm{C}_{2}, \mathrm{C}_{3}\right)$ obtidas em feiras livres. Uma vez coletadas foram imediatamente submetidas aos ensaios analíticos, em triplicatas.

\section{1 - Determinação de nitrito residual}

Foi determinado conforme o método desenvolvido por ARAÚJO \& MÍDIO [1], que se fundamenta na reação do íon nitroso $\left(\mathrm{NO}_{2}^{-}\right)$com composto aromático aminado (sulfanilamida), em meio ácido, para formar um sal de diazônio, seguido da adição de um composto aromático dicloridrato de $\mathrm{n}$ - (1-naftil) etilenodiamina que resulta na formação de pigmento azóico róseo, detectado em espectrofotômetro marca BECKMAN, modelo DUR-62, ao comprimento de onda de 538nm.

\section{2 - Determinação de nitrato}

O nitrato foi reduzido a nitrito, após passagem em coluna de cádmio/cobre e dosado como nitrito [1].

\section{3 - Análise estatística}

Foram aplicados testes ANOVA e Tukey, a nivel de $5 \%$ de significância, utilizando-se software STATISTICS FOR WINDOWS, versão 5.0 [17]

\section{3 - RESULTADOS E DISCUSSÃO}

\section{1 - Nitrito residual}

Os teores de nitrito nas salsichas comercializadas na Região Metropolitana do Recife encontram-se representados na Tabela 1.

TABELA 1. Nitrito residual (mg/kg) em salsichas comercializadas na região metropolitana do Recife-PE, 2001

\begin{tabular}{cccccccccc}
\hline $\begin{array}{c}\text { GRUPOS DE } \\
\text { INDÚSTRIAS }\end{array}$ & \multicolumn{3}{c}{$\mathrm{A}$} & \multicolumn{5}{c}{$\mathrm{B}$} \\
\hline MARCAS & $\mathrm{A}_{1}$ & $\mathrm{~A}_{2}$ & $\mathrm{~A}_{3}$ & $\mathrm{~B}_{1}$ & $\mathrm{~B}_{2}$ & $\mathrm{~B}_{3}$ & $\mathrm{C}_{1}$ & $\mathrm{C}_{2}$ & $\mathrm{C}_{3}$ \\
\hline & 27,50 & 32,00 & 36,00 & 120,0 & 76,00 & 101,5 & 153,1 & 115,2 & 155,3 \\
& 76,50 & 34,41 & 45,00 & 102,0 & 95,00 & 90,40 & 139,1 & 162,1 & 157,7 \\
$\mathrm{NO}_{2}^{-}$ & 38,40 & 70,50 & 70,10 & 95,40 & 70,80 & 72,00 & 90,00 & 107,2 & 135,4 \\
$(\mathrm{mg} / \mathrm{Kg})$ & 70,00 & 49,00 & 55,15 & 72,00 & 56,75 & 52,30 & 152,1 & 150,4 & 180,2 \\
& 54,00 & 51,00 & 39,32 & 49,20 & 69,32 & 72,50 & 110,7 & 180,1 & 142,7 \\
& 39,50 & 31,00 & 42,60 & 97,50 & 82,60 & 90,80 & 104,2 & 159,3 & 172,0 \\
\hline MÉDIA* $^{*}$ & 59,98 & 44,65 & 48,02 & 89,28 & 75,07 & 79,92 & 124,8 & 145,7 & 157,2 \\
\pm DP & 19,30 & 15,35 & 12,62 & 24,94 & 12,97 & 17,75 & 26,77 & 28,53 & 16,93 \\
\hline MÉDIA & \multirow{2}{*}{$47,88 \pm 15,26^{\mathrm{A}}$} & & $81,42 \pm 19,02^{\mathrm{B}}$ & & $142,62 \pm 26,91^{\mathrm{C}}$ \\
GERAL ** & & & & & & & & & \\
\hline
\end{tabular}

* letras minúsculas diferentes na mesma linha indicam diferenças significativas $(p<0,05)$. ** letras maiúsculas diferentes na mesma linha indicam diferenças significativas ( $(\mathrm{p}<0,05)$.

Os valores médios de nitrito encontrado nas salsichas das indústrias do Sul, do Nordeste e das feiras livres, variaram estatisticamente $(\mathrm{p}<0,05)$ de $47,88 \mathrm{mg} / \mathrm{kg}$ $( \pm 15,26)$, no grupo A, até $142 \mathrm{mg} / \mathrm{kg}( \pm 26,91)$, no grupo C. As amostras produzidas pelas indústrias do Sul embora tenham diferido $(\mathrm{p}<0,05)$ de todas provenientes das feiras livres, não apresentaram diferença em relação às produzidas pelas indústrias $\mathrm{B}_{2}$ e $\mathrm{B}_{3}$ da região Nordeste. Os valores entre as diferentes indústrias, de um mesmo grupo, foram homogêneos (para todos os grupos). Apenas nas salsichas das feiras livres (grupo C) constatou-se que $67 \%$ que representa $18 \%$ do total geral das amostras, apresentaram valores acima de $150 \mathrm{mg} / \mathrm{kg}$, máximo permitido pela legislação brasileira vigente, Portaria no 1004 de 11 de dezembro de 1998 [3], que restringe ainda mais o uso desses sais em relação à legislação anterior que estabelecia um limite máximo de $200 \mathrm{mg} / \mathrm{kg}$ de nitrito residual [2]. Esses dados diferem dos de CASSENS [4], que encontrou valores de nitrito residual entre 5 e 15mg/kg em produtos cárneos curados.

\section{2 - Nitrato (expresso em $\mathrm{mg} / \mathrm{kg}$ de $\mathrm{NaNO}_{2}$ )}

Os teores de nitrato (expressos em mg/kg de $\mathrm{NaNO}_{2}$ ) das salsichas comercializadas na Região Metropolitana do Recife estão explicitados na Tabela 2. 
TABELA 2. Teores de nitrato (expressos em mg/kg $\mathrm{NaNO}_{2}$ ) nas salsichas comercializadas na região metropolitana do Recife-PE, 2001

\begin{tabular}{|c|c|c|c|c|c|c|c|c|c|}
\hline \multirow{2}{*}{$\begin{array}{l}\text { GRUPOS DE } \\
\text { INDÚSTRIAS } \\
\text { MARCAS }\end{array}$} & \multicolumn{3}{|c|}{ A } & \multicolumn{4}{|c|}{ B } & \multicolumn{2}{|c|}{ C } \\
\hline & $A_{1}$ & $\mathrm{~A}_{2}$ & $\mathrm{~A}_{3}$ & $\mathrm{~B}_{1}$ & $\mathrm{~B}_{2}$ & $\mathrm{~B}_{3}$ & $\mathrm{C}_{1}$ & $\mathrm{C}_{2}$ & $\mathrm{C}_{3}$ \\
\hline \multirow{6}{*}{$\begin{array}{c}\mathrm{NO}_{3}^{-} \\
(\mathrm{mg} / \mathrm{Kg})\end{array}$} & 138,4 & 72,00 & 191,4 & 305,2 & 187,0 & 156,4 & 420,2 & 320,4 & 327,1 \\
\hline & 103,8 & 62,50 & 310,2 & 325,0 & 190,4 & 304,9 & 280,4 & 380,3 & 292,1 \\
\hline & 78,53 & 80,42 & 270,0 & 297,0 & 315,0 & 329,4 & 315,7 & 470,1 & 335,1 \\
\hline & 109,4 & 65,30 & 315,0 & 285,0 & 287,4 & 335,7 & 342,1 & 335,2 & 435,1 \\
\hline & 115,2 & 71,42 & 329,4 & 401,0 & 311,0 & 420,3 & 482,1 & 270,2 & 337,9 \\
\hline & 121,9 & 70,55 & 178,4 & 407,1 & 314,5 & 410,0 & 475,3 & 310,1 & 482,1 \\
\hline MÉDIA* & 111,2 & 70,36 & 265,7 & 336,7 & 267,5 & 326,1 & 386,0 & 347,7 & 368,2 \\
\hline$\pm \mathrm{DP}$ & 19,99 & 6,20 & 65,74 & 53,78 & 61,93 & 95,13 & 85,34 & 69,83 & 73,40 \\
\hline $\begin{array}{c}\text { MÉDIA } \\
\text { GERAL** }\end{array}$ & \multicolumn{3}{|c|}{$149,11 \pm 94,32^{A}$} & \multicolumn{3}{|c|}{$310,14 \pm 74,97^{\mathrm{B}}$} & \multicolumn{3}{|c|}{$367,35 \pm 73,61^{B}$} \\
\hline
\end{tabular}

Os valores médios de nitrito + nitrato (expressos em $\mathrm{mg} / \mathrm{kg} \mathrm{NaNO}_{2}$ ) demonstram que houve variação de $149,11 \mathrm{mg} / \mathrm{kg}( \pm 94,32)$, no grupo A, até $367,35 \mathrm{mg} / \mathrm{kg}$ $( \pm 73,61)$, no grupo $C$, com diferença significativa $(\mathrm{p}<0,05)$ entre as médias das amostras da região Sul (A) e os demais grupos, destacando-se valores extremos de $78,53 \mathrm{mg} / \mathrm{kg}$ indústria $\left(\mathrm{A}_{1}\right)$ a $482,19 \mathrm{mg} / \mathrm{kg}$ indústria $\left(\mathrm{C}_{1}\right)$. Nas representantes de um mesmo grupo, houve variação significativa apenas entre a indústria $A_{3}$ e as indústrias $A_{1}$ e $A_{2}$, essas, as únicas que variaram significativamente de todos os outros sub-grupos de indústrias. Segundo a legislação anterior [2] à vigente, $100 \%$ das amostras estão abaixo dos limites legais uma vez que aquela estabelecia um máximo de $500 \mathrm{mg} / \mathrm{kg}$ de nitrato de sódio ou potássio, combinado ou não com nitrito. Porém, considerando a atual [3] que determina um máximo de $300 \mathrm{mg} / \mathrm{kg}$ de nitrato/100g do produto acabado, constatou-se que 56\% do total geral das amostras estavam em desacordo, especificamente, $17 \%$ das amostras do grupo A, 67\% do grupo B e $83 \%$ do grupo C.

Estes resultados discordam dos obtidos por LARA, TAKAHASHI, SILVEIRA ([10] que, analisando 7 marcas de salsichas comercializadas na Região Metropolitana de São Paulo, encontraram níveis de nitrato (expressos em $\mathrm{NaNO}_{2}$ ) inferiores a mg/kg. Em contrapartida, SOUZA, FALEIROS, SOUZA [16] determinaram um teor máximo de $282 \mathrm{mg} / \mathrm{kg}$ de nitrato (expresso em $\mathrm{NaNO}_{2}$ ) em salsichas comercializadas em supermercados, valor inferior ao valor máximo encontrado neste trabalho.

\section{4 - CONCLUSÕES}

As salsichas comercializadas na Região Metropolitana do Recife apresentam índices comprometedores de nitrato, principalmente, as das indústrias que abastecem as feiras livres, fato agravado pelos altos níveis de nitrito também presente.

\section{5 - REFERÊNCIAS BIBLIOGRÁFICAS}

[1] ARAÚJO, J.M.; MÍDIO, A.F. Determinação espectrofotométrica de nitritos e nitratos após redução com coluna de cádmio/cobre em alimentos destinados à população infantil. Revista de Farmácia e Bioquímica da Universidade de São Paulo, v. 25, n. 1, p. 570, 1989.

[2] BRASIL. Decreto Federal no 1255, de 25. 06.1962. Dispõe sobre regulamentos de inspeção industrial e sanitária de produtos de origem animal. Brasília, Ministério da Agricultura, 1962.

[3] BRASIL. Portaria no 1004, de 11. 12. 1998. Brasília, Ministério da Saúde, 1999.

[4] CASsens, R.G. Residual nitrite in cured meat. Food Technology, v. 51, n. 2, p. 53-55, 1997.

[5] EICHHOLZER, M.; GUTZWILLER, F. Dietary nitrates, nitrites, and N-nitroso compounds and cancer risk: a review of the epidemiologic evidence. Nutrition Reviews, v. 56, n. 4, p.95-105, 1998.

[6] FERREIRA, S.M.R.; CAMARGO, L. Aditivos em alimentos. Boletim do CEPPA, v. 11, n. 2, p. 159-176, 1993.

[7] GONÇALVES, J.R. Qualidade e competitividade; requisitos a industrialização de salsicha. Revista Nacional da Carne, n. 220, p. 39-42, 1995.

[8] HILL, M.J. Nitrate toxicity: myth or reality. British Journal of Nutrition, v. 81, p. 343-344, 1999.

[9] KOMATSU, I.; TAKINO, M.; GALLI, F. O teor de nitrito e nitrato nos produtos cárneos fabricados no estado de São Paulo. Ciência Cultural, v. 29, p.124-126, 1977.

[10] LARA, W.H.; TAKAHASHI, M.Y.; SILVEIRA, N. Determinação de nitritos e nitratos em conservas de carne. Revista do Instituto Adolfo Lutz, v. 38, p. 161-166, 1978.

[11] McKNIGHT, G.M.; DUCAN, C.W.; LEIFERT, C.; GPLDEN, M.H. Dietary nitrate in man: friend or foe?. British Journal of Nutrition, n. 81, p. 349-358, 1999.

[12] MELO FILHO, A.B.; GUERRA, N.B. Avaliação da qualidade nutricional de produtos cárneos: salsichas e mortadelas comercializadas na Região Metropolitana do Recife. In: CONGRESSO LATINOAMERICANO DE NUTRICIONISTAS-DIETISTAS, Montevideo. 1998. p. 186.

[13] PÉREZ-RODRIGUEZ, M.L.; BOSCH-BOSCH, N.; GARCIÁ-MATA, M. Monitoring nitrite and nitrate residues in frankfurters during processing and storage. Meat Science, v. 44, n. 1, p. 65-73, 1996.

[14] SILVA, J.A. Ocorrência e controle de clostridium botulinum em produtos cárneos curados. Revista Tecnológica e Ciências de Carnes, v. 1, n. 1, p. 44-56, 1999.

[15] SILVA, P. da. O mercado de embutidos cresce no Brasil. Alimentos e Tecnologia, v. 61 , n. 10, p. 4041,1995 .

[16] SOUZA, P.A.; FALEIROS, R.R. S.; SOUZA, H.B.A. Dosagem de nitrito e nitrato em produtos embutidos de carne. Alimentação e Nutrição, São Paulo, v. 2, p. 2734, 1990.

[17] STATSOFT. Statistic for windows, (Computer program manual). Tulsa, 1996.

[18] TERRA, N.N. A cor nos produtos cárneos. Revista Nacional da Carne, n. 269, p. 35-42, jul. 1999.

[19] TORRE, J.C.M.D.; RODRIGUES, R.S.M. Sais de cura em carne: legislação da Alemanha e do Brasil. Revista Nacional da Carne, n. 257, p. 16-20, jul. 1998. 